Georgian Mathematical Journal

Volume 14 (2007), Number 1, 53-64

\title{
THE MONOMIALITY APPROACH TO MULTI-INDEX POLYNOMIALS IN SEVERAL VARIABLES
}

\author{
CARLO BELINGERI, GIUSEPPE DATTOLI, AND PAOLO E. RICCI
}

Dedicated to the Memory of Prof. Gaetano Fichera

\begin{abstract}
The monomiality principle was introduced (see [1] and the references therein) in order to derive the properties of special or generalized polynomials starting from the corresponding ones of monomials. We show a general technique of extending the monomiality approach to multi-index polynomials in several variables. Application of this technique to the case of Hermite, Laguerre-type and mixed-type (i.e., between Laguerre and Hermite) polynomials is given.
\end{abstract}

2000 Mathematics Subject Classification: 44A45, 30D05, 33C45.

Key words and phrases: Exponential operators, operational calculus, monomiality principle, Gould-Hopper polynomials, multi-index Hermite and Laguerre polynomials.

\section{INTRODUCTION}

In the preceding articles [2], [3], the monomiality technique (see [1] and the references therein) was used to introduce general polynomial sets in several variables, including the Hermite-Kampé de Fériet or Gould-Hopper polynomials [4], [5], [6], and new sets of Laguerre-type polynomials.

Although in the early 40s of the last century J. F. Steffensen [7] suggested the concept of poweroid, it is only recently that the concept of monomiality has shown all its importance and flexibility (see [1]).

Y. Ben Cheikh proved in refs. [8], [9] that all polynomial families can be viewed as quasi-monomials with respect to a suitably defined derivative and multiplication operators, but in general such operators are expressed by formal series of the derivative so that it is impossible to obtain formulas sufficiently simple to handle. However, for particular polynomials sets related to suitable generating functions the above-mentioned formal series reduce to finite sums, so that the relevant properties can be easily derived (see, e.g., [10], [11], [12], [13]).

In this paper, by employing the two-variable monomiality technique, we derive some two-index polynomial sets, and we extend the results obtained in [2], [3], introducing the many-variable two-index Hermite, Laguerre-type and mixed-type (i.e., composed by both Hermite and Laguerre) polynomials.

The results are easily extended to the case of many-variable many-index polynomials. A brief survey in this direction is presented in the last Section. 


\section{The Two-Variable Monomiality Principle}

The two-variable monomiality principle is defined as follows.

Definition 2.1. Let $\left\{p_{n, m}(x, y)\right\}_{(n, m) \in \mathbf{N}_{0} \times \mathbf{N}_{0}}\left(\mathbf{N}_{0}:=\mathbf{N} \cup 0\right)$ be a two-variable two-index polynomial set with $\operatorname{deg} p_{n, m}(\cdot, y)=n$ and $\operatorname{deg} p_{n, m}(x, \cdot)=m$.

$\left\{p_{n, m}(x, y)\right\} \equiv\left\{p_{n, m}(x, y)\right\}_{(n, m) \in \mathbf{N}_{0} \times \mathbf{N}_{0}}$ is said to be quasi-monomial if four operators, not depending on $n$ and $m$, denoted by $\hat{P}_{x}, \hat{P}_{y}, \hat{M}_{x}, \hat{M}_{y}$, exist in such a way that

$$
\begin{gathered}
\left\{\begin{array}{l}
\hat{P}_{x} p_{n, m}(x, y)=n p_{n-1, m}(x, y), \\
\hat{P}_{y} p_{n, m}(x, y)=m p_{n, m-1}(x, y),
\end{array}\right. \\
\left\{\begin{array}{l}
\hat{M}_{x} p_{n, m}(x, y)=p_{n+1, m}(x, y), \\
\hat{M}_{y} p_{n, m}(x, y)=p_{n, m+1}(x, y) .
\end{array}\right.
\end{gathered}
$$

Note that the commutation properties

$$
\left[\hat{P}_{x}, \hat{M}_{x}\right]=1, \quad\left[\hat{P}_{y}, \hat{M}_{y}\right]=1
$$

follow from equations $(2.1),(2.2)$, so that the above operators display Weyl group structures.

Under the above hypotheses, the main properties of the polynomial set under consideration can be easily derived, since

- If the operators $\hat{P}_{x}, \hat{P}_{y}, \hat{M}_{x}, \hat{M}_{y}$ have a differential realization, then we find

$$
\hat{M}_{x} \hat{P}_{x} p_{n, m}(x, y)=n p_{n, m}(x, y), \quad \hat{M}_{y} \hat{P}_{y} p_{n, m}(x, y)=m p_{n, m}(x, y),
$$

i.e., two (independent) differential equations satisfied by the polynomial set.

- Assuming $p_{0,0}(x, y) \equiv 1$, the explicit expression of $\left\{p_{n, m}(x, y)\right\}$ is given by

$$
p_{n, m}(x, y)=\hat{M}_{x}^{n} \hat{M}_{y}^{m}(1) .
$$

- The exponential generating function of $\left\{p_{n, m}(x, y)\right\} \quad\left(p_{0,0}(x, y) \equiv 1\right)$ is given by

$$
\begin{aligned}
e^{t \hat{M}_{x}+\tau \hat{M}_{y}}(1) & =\sum_{n=0}^{\infty} \sum_{m=0}^{\infty} \frac{\left(t \hat{M}_{x}\right)^{n}\left(\tau \hat{M}_{y}\right)^{m}}{n ! m !} \\
& =\sum_{n=0}^{\infty} \sum_{m=0}^{\infty} \frac{t^{n}}{n !} \frac{\tau^{m}}{m !} p_{n, m}(x, y)
\end{aligned}
$$

\section{A Quasi-Monomiality Criterion}

In this section, we extend a result given in [2] to two-variable two-index polynomial sets. 
We start from a quasi-monomial set $\left\{p_{n, m}(x, y)\right\}$, and suppose to know the relevant derivative operators $\hat{P}_{x}, \hat{P}_{y}$. Then we derive a monomiality criterion for a four-variable two-index polynomial set.

Theorem 3.1. Consider two operators $\hat{\Phi}_{x}$ and $\hat{\Psi}_{y}$ commuting respectively with $\hat{P}_{x}$ and $\hat{P}_{y}$ and such that

$$
e^{z \hat{\Phi}_{x}+t \hat{\Psi}_{y}}(1)=1 .
$$

Put

$$
Q_{n, m}(x, y ; z, t):=e^{z \hat{\Phi}_{x}+t \hat{\Psi}_{y}} p_{n, m}(x, y),
$$

and suppose that there exist two operators $\hat{M}_{1, x}(z, t) \hat{M}_{1, y}(z, t)$ such that

$$
Q_{n, m}(x, y ; z, t)=\left(\hat{M}_{1, x}(z, t)\right)^{n}\left(\hat{M}_{1, y}(z, t)\right)^{m}
$$

and, furthermore, $\forall z, t$

$$
\left[\hat{P}_{x}, \hat{M}_{1, x}(z, t)\right]=\left[\hat{P}_{y}, \hat{M}_{1, y}(z, t)\right]=1 .
$$

Then the polynomial set $Q_{n, m}(x, y ; z, t)$ is quasi-monomial with respect to the operators $\hat{P}_{x}, \hat{P}_{y}, \hat{M}_{1, x}(z, t), \hat{M}_{1, y}(z, t)$.

Proof. Indeed, since $\hat{P}_{x}$ is commuting with $\hat{\Phi}_{x}$, and $\hat{\Psi}_{y}$ it is also commuting with $e^{z \hat{\Phi}_{x}+t \hat{\Psi}_{y}}$, we have

$$
\begin{aligned}
\hat{P}_{x}\left[Q_{n, m}(x, y ; z, t)\right] & =e^{z \hat{\Phi}_{x}+t \hat{\Psi}_{y}} \hat{P}_{x}\left[p_{n, m}(x, y)\right]=n e^{z \hat{\Phi}_{x}+t \hat{\Psi}_{y}} p_{n-1, m}(x, y) \\
& =n Q_{n-1, m}(x, y ; z, t) .
\end{aligned}
$$

Analogously, we find

$$
\hat{P}_{y}\left[Q_{n, m}(x, y ; z, t)\right]=m Q_{n, m-1}(x, y ; z, t) .
$$

Furthermore we obviously have

$$
\begin{array}{r}
\hat{M}_{1, x}(z, t)\left[Q_{n, m}(x, y ; z, t)\right]=\hat{M}_{1, x}(z, t)\left(\hat{M}_{1, x}(z, t)\right)^{n}\left(\hat{M}_{1, y}(z, t)\right)^{m}(1) \\
=\left(\hat{M}_{1, x}(z, t)\right)^{n+1}\left(\hat{M}_{1, y}(z, t)\right)^{m}(1)=Q_{n+1, m}(x, y ; z, t)
\end{array}
$$

and

$$
\begin{array}{r}
\hat{M}_{1, y}(z, t)\left[Q_{n, m}(x, y ; z, t)\right]=\hat{M}_{1, y}(z, t)\left(\hat{M}_{1, x}(z, t)\right)^{n}\left(\hat{M}_{1, y}(z, t)\right)^{m}(1) \\
=\left(\hat{M}_{1, x}(z, t)\right)^{n}\left(\hat{M}_{1, y}(z, t)\right)^{m+1}(1)=Q_{n, m+1}(x, y ; z, t) .
\end{array}
$$

The construction of the operators $\hat{M}_{1, x}(z, t)$ and $\hat{M}_{1, y}(z, t)$, starting from the multiplication operators $\hat{M}_{x}, \hat{M}_{y}$ of the basic set $\left\{p_{n, m}(x, y)\right\}$, can be performed by using the two-variable Hausdorff identity presented in Section 4. 


\section{A Two-Variable Hausdorff Identity}

A two-variable Hausdorff identity can be derived as follows

Theorem 4.1. Let $\hat{A}$ and $\hat{B}$, be two commuting operators (i.e., $[\hat{A}, \hat{B}]=0$ ) independent of the parameters $z$ and $t$. Then the Hausdorff identity holds:

$$
\begin{aligned}
e^{z \hat{A}+t \hat{B}} \hat{C} e^{-z \hat{A}-t \hat{B}}= & \hat{C}+(z[\hat{A}, \hat{C}]+t[\hat{B}, \hat{C}]) \\
& +\frac{1}{2 !}\left(z^{2}[\hat{A},[\hat{A}, \hat{C}]]+2 z t[\hat{A},[\hat{B}, \hat{C}]]+t^{2}[\hat{B},[\hat{B}, \hat{C}]]\right) \\
& +\frac{1}{3 !}\left(z^{3}[\hat{A},[\hat{A},[\hat{A}, \hat{C}]]]+3 z^{2} t[\hat{A},[\hat{A},[\hat{B}, \hat{C}]]]\right. \\
& \left.+3 z t^{2}[\hat{A},[\hat{B},[\hat{B}, \hat{C}]]]+t^{3}[\hat{B},[\hat{B},[\hat{B}, \hat{C}]]]\right)+\cdots
\end{aligned}
$$

Proof. We proceed in a similar way with respect to the classical case [14]. The above identity can be derived by using the Taylor expansion with respect to the parameters $z$ and $t$ :

$$
e^{z \hat{A}+t \hat{B}} \hat{C} e^{-z \hat{A}-t \hat{B}}=\left.\sum_{k=0}^{\infty} \frac{1}{k !} d^{k}\left(e^{z \hat{A}+t \hat{B}} \hat{C} e^{-z \hat{A}-t \hat{B}}\right)\right|_{z=t=0} .
$$

From the commuting property of $\hat{A}$ and $\hat{B}$, it follows that they commute even with $e^{z \hat{A}+t \hat{B}}$ and $e^{-z \hat{A}-t \hat{B}}$. Furthermore, the identity $[\hat{A},[\hat{B}, \hat{C}]]+[\hat{C},[\hat{A}, \hat{B}]]+$ $[\hat{B},[\hat{C}, \hat{A}]]=0$ implies $[\hat{A},[\hat{B}, \hat{C}]]=[\hat{B},[\hat{A}, \hat{C}]]$ so that the coefficients of all mixed derivatives with respect to $z$ and $t$ must coincide.

4.1. A special case of Equation (4.1). Consider first the identity

$$
e^{t\left(D_{x}^{2}+D_{y}^{2}\right)} x^{p} y^{q}=e^{t\left(D_{x}^{2}+D_{y}^{2}\right)} x^{p} y^{q} e^{-t\left(D_{x}^{2}+D_{y}^{2}\right)}(1) .
$$

Iterating the above equation $p$ times with respect to $x$ and $q$ times with respect to $y$, and denoting by $H_{n}(x, y)$ the Hermite-Kampé de Fériet polynomials, we obtain

$$
\begin{gathered}
e^{t\left(D_{x}^{2}+D_{y}^{2}\right)} x^{p} y^{q}=e^{t\left(D_{x}^{2}+D_{y}^{2}\right)} x e^{-t\left(D_{x}^{2}+D_{y}^{2}\right)} \cdots e^{t\left(D_{x}^{2}+D_{y}^{2}\right)} x e^{-t\left(D_{x}^{2}+D_{y}^{2}\right)} \\
\times e^{t\left(D_{x}^{2}+D_{y}^{2}\right)} y e^{-t\left(D_{x}^{2}+D_{y}^{2}\right)} \cdots e^{t\left(D_{x}^{2}+D_{y}^{2}\right)} y e^{-t\left(D_{x}^{2}+D_{y}^{2}\right)}(1) \\
=\left(x+2 t D_{x}\right)^{p}\left(y+2 t D_{y}\right)^{q}(1) \\
=\left(x+2 t D_{x}\right)^{p} H_{q}(y, t)=\sum_{k=0}^{p} H_{p-k}(x, t)\left(2 t D_{x}\right)^{k} H_{q}(y, t) \\
=H_{p}(x, t) H_{q}(y, t),
\end{gathered}
$$

since in the last summation the only nonvanishing term corresponds to the index $k=0$.

More generally, by introducing the Gould-Hopper polynomials $H_{n}^{(m)}(x, y)$, for any integer numbers $m, n$ we can write

$$
e^{t\left(D_{x}^{m}+D_{y}^{n}\right)} x^{p} y^{q}=\left(x+m t D_{x}^{m-1}\right)^{p}\left(y+n t D_{y}^{n-1}\right)^{q}(1)=H_{p}^{(m)}(x, t) H_{q}^{(n)}(y, t) .
$$




\section{Construction of Multiplication Operators}

Recalling the method developed in [3], and using the two-variable Hausdorff identity of the preceding section, we can construct the multiplication operators $\hat{M}_{1, x}(z, t) \hat{M}_{1, y}(z, t)$, starting from the same operators $\hat{M}_{x}, \hat{M}_{y}$ of the basic set $\left\{p_{n, m}(x, y)\right\}$.

Theorem 5.1. Consider the polynomial set $\left\{p_{n, m}(x, y)\right\}$ with $p_{0,0}(x, y) \equiv$ 1 and suppose that it is quasi-monomial with respect to the operators $\hat{P}_{x}, \hat{P}_{y}$, $\hat{M}_{x}, \hat{M}_{y}$. Let $\hat{\Phi}_{x}$ and $\hat{\Psi}_{y}$, be two operators, independent of the parameters $z$ and $t$, such that

$$
\left[\hat{\Phi}_{x}, \hat{P}_{x}\right]=\left[\hat{\Psi}_{y}, \hat{P}_{y}\right]=0, \quad e^{z \hat{\Phi}_{x}+t \hat{\Psi}_{y}}(1)=1 .
$$

For the polynomial set defined as

$$
Q_{n, m}(x, y ; z, t):=e^{z \hat{\Phi}_{x}+t \hat{\Psi}_{y}} p_{n, m}(x, y),
$$

the derivative operators are given by $\hat{P}_{1, x}=\hat{P}_{x}, \hat{P}_{1, y}=\hat{P}_{y}$ (Theorem 3.1 ).

Furthermore, the multiplication operators are given by the Hausdorff expansions

$$
\begin{aligned}
\hat{M}_{1, x}= & \hat{M}_{x}+\left\{z\left[\hat{\Phi}_{x}, \hat{M}_{x}\right]+t\left[\hat{\Psi}_{y}, \hat{M}_{x}\right]\right\}+\frac{1}{2 !}\left\{z^{2}\left[\hat{\Phi}_{x},\left[\hat{\Phi}_{x}, \hat{M}_{x}\right]\right]\right. \\
& \left.+2 z t\left[\hat{\Phi}_{x},\left[\hat{\Psi}_{y}, \hat{M}_{x}\right]\right]+t^{2}\left[\hat{\Psi}_{y},\left[\hat{\Psi}_{y}, \hat{M}_{x}\right]\right]\right\}+\cdots, \\
\hat{M}_{1, y}= & \hat{M}_{y}+\left\{z\left[\hat{\Phi}_{x}, \hat{M}_{y}\right]+t\left[\hat{\Psi}_{y}, \hat{M}_{y}\right]\right\}+\frac{1}{2 !}\left\{z^{2}\left[\hat{\Phi}_{x},\left[\hat{\Phi}_{x}, \hat{M}_{y}\right]\right]\right. \\
& \left.+2 z t\left[\hat{\Phi}_{x},\left[\hat{\Psi}_{y}, \hat{M}_{y}\right]\right]+t^{2}\left[\hat{\Psi}_{y},\left[\hat{\Psi}_{y}, \hat{M}_{y}\right]\right]\right\}+\cdots .
\end{aligned}
$$

Some examples easy to handle, since the above expansions reduce to finite sums, will be shown in the next section.

Proof. From equation (2.5) we have $p_{n, m}(x, y)=\hat{M}_{x}^{n} \hat{M}_{y}^{m}(1)$ and, consequently, recalling (3.1), we can write

$$
Q_{n, m}(x, y ; z, t)=e^{z \hat{\Phi}_{x}+t \hat{\Psi}_{y}} \hat{M}_{x}^{n} \hat{M}_{y}^{m}(1) .
$$

Applying the Hausdorff identity of Theorem 4.1 and using (5.1), we find

$$
\begin{aligned}
e^{z \hat{\Phi}_{x}+t \hat{\Psi}_{y}} \hat{M}_{x} & =e^{z \hat{\Phi}_{x}+t \hat{\Psi}_{y}} \hat{M}_{x} e^{-z \hat{\Phi}_{x}-t \hat{\Psi}_{y}}(1) \\
& =\hat{M}_{x}+\left\{z\left[\hat{\Phi}_{x}, \hat{M}_{x}\right]+t\left[\hat{\Psi}_{y}, \hat{M}_{x}\right]\right\}+\cdots=: \hat{M}_{1, x}(z, t),
\end{aligned}
$$

since

$$
\begin{aligned}
Q_{n, 0}(x, y ; z, t)= & e^{z \hat{\Phi}_{x}+t \hat{\Psi}_{y}} \hat{M}_{x}^{n}(1) \\
= & e^{z \hat{\Phi}_{x}+t \hat{\Psi}_{y}} \hat{M}_{x} e^{-z \hat{\Phi}_{x}-t \hat{\Psi}_{y}} e^{z \hat{\Phi}_{x}+t \hat{\Psi}_{y}} \hat{M}_{x} e^{-z \hat{\Phi}_{x}-t \hat{\Psi}_{y}} \\
& \times \cdots \times e^{z \hat{\Phi}_{x}+t \hat{\Psi}_{y}} \hat{M}_{x} e^{-z \hat{\Phi}_{x}-t \hat{\Psi}_{y}}(1)=\left(\hat{M}_{1, x}(z, t)\right)^{n}(1) .
\end{aligned}
$$


Analogously,

$$
\begin{aligned}
e^{z \hat{\Phi}_{x}+t \hat{\Psi}_{y}} \hat{M}_{y} & =e^{z \hat{\Phi}_{x}+t \hat{\Psi}_{y}} \hat{M}_{y} e^{-z \hat{\Phi}_{x}-t \hat{\Psi}_{y}}(1) \\
& =\hat{M}_{y}+\left\{z\left[\hat{\Phi}_{x}, \hat{M}_{y}\right]+t\left[\hat{\Psi}_{y}, \hat{M}_{y}\right]\right\}+\cdots=: \hat{M}_{1, y}(z, t)
\end{aligned}
$$

so that

$$
Q_{n, m}(x, y ; z, t)=\left(\hat{M}_{1, x}(z, t)\right)^{n}\left(\hat{M}_{1, y}(z, t)\right)^{m}(1) .
$$

Therefore, recalling the monomiality criterion of Theorem 3.1, the multiplication operators of the polynomial set $Q_{n, m}(x, y ; z, t)$ are given by $\hat{M}_{1, x}(z, t)$ and $\hat{M}_{1, y}(z, t)$.

Remark 5.2. By applying inductively $r$-times the above technique, quasimonomial sets in $2(r+1)$ variables can be derived. Of course, even quasimonomial sets with an odd number of independent variables are included, since for example in equation (3.1) we could assume $t=0$ or $z=0$. Note that, assuming $t=z=1$, a new set of quasi-monomial polynomials is defined without increasing the number of independent variables.

\section{EXAmples}

In this section, writing the basic polynomial set as

$$
\begin{aligned}
p_{n, m}(x, y) & =x^{n} y^{m}, & & \text { or } \\
q_{n, m}(x, y) & =\frac{x^{n}}{n !} \frac{y^{m}}{m !}, & & \text { or } \\
r_{n, m}(x, y) & =x^{n} \frac{y^{m}}{m !} & &
\end{aligned}
$$

and, correspondingly, the derivative and multiplication operators as

$$
\begin{aligned}
\hat{P}_{x}=D_{x}, & \hat{P}_{y}=D_{y}, \\
\hat{M}_{x}=x \cdot & \hat{M}_{y}=y .
\end{aligned}
$$

or as

$$
\begin{gathered}
\hat{P}_{x}=D_{x} x D_{x}, \quad \hat{P}_{y}=D_{y} y D_{y}, \\
\hat{M}_{x}=\int_{0}^{x}, \quad \hat{M}_{y}=\int_{0}^{y}
\end{gathered}
$$

or as

$$
\begin{array}{ll}
\hat{P}_{x}=D_{x}, & \hat{P}_{y}=D_{y} y D_{y}, \\
\hat{M}_{x}=x \cdot, & \hat{M}_{y}=\int_{0}^{y},
\end{array}
$$

we find two-variable two-index polynomials of Hermite, Laguerre and mixedtype polynomials by considering suitable operators $\hat{\Phi}_{x}$ and $\hat{\Psi}_{y}$. 
It is worth to note that Laguerre and mixed-type polynomials can be derived by the Hermite ones by applying the differential isomorphism $\mathcal{T}$ described in [15], with respect to the variables $x$ or $y$. This isomorphism is denoted consequently by $\mathcal{T}_{x}$ or $\mathcal{T}_{y}$. Generalization to the case of two-variable, two-index polynomials of Gould-Hopper-type and their Laguerre counterpart, introduced in [15], can be constructed in a similar way.

6.1. The Hermite case. Let us fix the integers $j_{1}, j_{2}$, assume $p_{n, m}(x, y):=$ $x^{n} y^{m}$ and, moreover,

$$
\begin{array}{cc}
\hat{P}_{0, x} \equiv \hat{P}_{x}:=\frac{\partial}{\partial x}=D_{x}, & \hat{M}_{0, x}:=x \\
\hat{P}_{0, y} \equiv \hat{P}_{y}:=\frac{\partial}{\partial y}=D_{y}, & \hat{M}_{0, y}:=y \\
\hat{\Phi}_{x}:=\frac{\partial^{j_{1}}}{\partial x^{j_{1}}}, & \hat{\Psi}_{y}:=\frac{\partial^{j_{2}}}{\partial y^{j_{2}}} .
\end{array}
$$

Applying Theorem 5.1, we find

$$
\begin{aligned}
& \hat{M}_{1, x}=x+j_{1} z \frac{\partial^{j_{1}}}{\partial x^{j_{1}}} \\
& \hat{M}_{1, y}=y+j_{2} t \frac{\partial^{j_{2}}}{\partial y^{j_{2}}} .
\end{aligned}
$$

Therefore, since

$$
\begin{aligned}
& \exp \left(z \frac{\partial^{j_{1}}}{\partial x^{j_{1}}}\right) x^{n}=H_{n}^{\left(j_{1}\right)}(x, z)=\left(x+j_{1} z \frac{\partial^{j_{1}-1}}{\partial x^{j_{1}-1}}\right)^{n}(1), \\
& \exp \left(t \frac{\partial^{j_{2}}}{\partial y^{j_{2}}}\right) y^{m}=H_{m}^{\left(j_{2}\right)}(y, t)=\left(y+j_{2} t \frac{\partial^{j_{2}-1}}{\partial y^{j_{2}-1}}\right)^{m}(1)
\end{aligned}
$$

we have

$$
Q_{n, m}(x, y ; z, t)=\exp \left\{z \frac{\partial^{j_{1}}}{\partial x^{j_{1}}}+t \frac{\partial^{j_{2}}}{\partial y^{j_{2}}}\right\}(1)=H_{n}^{\left(j_{1}\right)}(x, z) H_{m}^{\left(j_{2}\right)}(y, t)
$$

6.2. The Laguerre case. Considering again the integers $j_{1}, j_{2}$, assume $p_{n, m}(x, y):=\left(x^{n} / n !\right)\left(y^{m} / m !\right)$ and, moreover,

$$
\begin{array}{ll}
\hat{P}_{0, x} \equiv \hat{P}_{x}:=\left(D_{L}\right)_{x}=D_{x} x D_{x}, & \hat{M}_{0, x}:=\hat{D}_{x}^{-1}, \\
\hat{P}_{0, y} \equiv \hat{P}_{y}:=\left(D_{L}\right)_{y}=D_{y} y D_{y}, & \hat{M}_{0, y}:=\hat{D}_{y}^{-1}, \\
\hat{\Phi}_{x}:=\left(D_{L}\right)_{x}^{j_{1}}=D_{x}^{j_{1}} x^{j_{1}} D_{x}^{j_{1}}, & \hat{\Psi}_{y}:=\left(D_{L}\right)_{y}^{j_{2}}=D_{y}^{j_{2}} y^{j_{2}} D_{y}^{j_{2}} .
\end{array}
$$

Applying Theorem 5.1, we find

$$
\begin{aligned}
& \hat{M}_{1, x}=\hat{D}_{x}^{-1}+j_{1} z\left(D_{L}\right)_{x}^{j_{1}-1}, \\
& \hat{M}_{1, y}=\hat{D}_{y}^{-1}+j_{2} t\left(D_{L}\right)_{y}^{j_{2}-1} .
\end{aligned}
$$


Therefore, since

$$
\begin{aligned}
& \exp \left(z\left(D_{L}\right)_{x}^{j_{1}}\right) \frac{x^{n}}{n !}=L_{n}^{\left(j_{1}\right)}(x, z)=\left(\hat{D}_{x}^{-1}+j_{1} z\left(D_{L}\right)_{x}^{j_{1}-1}\right)^{n}(1), \\
& \exp \left(t\left(D_{L}\right)_{y}^{j_{2}}\right) \frac{y^{m}}{m !}=L_{m}^{\left(j_{2}\right)}(y, t)=\left(\hat{D}_{y}^{-1}+j_{2} t\left(D_{L}\right)_{y}^{j_{2}-1}\right)^{m}(1)
\end{aligned}
$$

we have

$$
Q_{n, m}(x, y ; z, t)=\exp \left\{z\left(D_{L}\right)_{x}^{j_{1}}+t\left(D_{L}\right)_{y}^{j_{2}}\right\}(1)=L_{n}^{\left(j_{1}\right)}(x, z) L_{m}^{\left(j_{2}\right)}(y, t) .
$$

6.3. The mixed case. Let us fix the integers $j_{1}, j_{2}$, assume $p_{n, m}(x, y):=$ $x^{n}\left(y^{m} / m !\right)$ and, moreover,

$$
\begin{gathered}
\hat{P}_{0, x} \equiv \hat{P}_{x}:=D_{x}, \quad \hat{M}_{0, x}:=x \cdot, \\
\hat{P}_{0, y} \equiv \hat{P}_{y}:=\left(D_{L}\right)_{y}=D_{y} y D_{y}, \quad \hat{M}_{0, y}:=\hat{D}_{y}^{-1}, \\
\hat{\Phi}_{x}:=D_{x}^{j_{1}}, \quad \hat{\Psi}_{y}:=\left(D_{L}\right)_{y}^{j_{2}}=D_{y}^{j_{2}} y^{j_{2}} D_{y}^{j_{2}} .
\end{gathered}
$$

Applying Theorem 5.1, we find

$$
\begin{aligned}
& \hat{M}_{1, x}=x+j_{1} z D_{x}^{j_{1}-1} \\
& \hat{M}_{1, y}=\hat{D}_{y}^{-1}+j_{2} t\left(D_{L}\right)_{y}^{j_{2}-1} .
\end{aligned}
$$

Therefore, since

$$
\begin{aligned}
\exp \left(z D_{x}^{j_{1}}\right) x^{n} & =H_{n}^{\left(j_{1}\right)}(x, z)=\left(x+j_{1} z D_{x}^{j_{1}-1}\right)^{n}(1), \\
\exp \left(t\left(D_{L}\right)_{y}^{j_{2}}\right) \frac{y^{m}}{m !} & =L_{m}^{\left(j_{2}\right)}(y, t)=\left(\hat{D}_{y}^{-1}+j_{2} t\left(D_{L}\right)_{y}^{j_{2}-1}\right)^{m}(1),
\end{aligned}
$$

we have

$$
Q_{n, m}(x, y ; z, t)=\exp \left\{z D_{x}^{j_{1}}+t\left(D_{L}\right)_{y}^{j_{2}}\right\}(1)=H_{n}^{\left(j_{1}\right)}(x, z) L_{m}^{\left(j_{2}\right)}(y, t) .
$$

\section{The $r$-Variable Monomiality Principle}

In this section we give the main results of this paper, omitting the proofs, since the technique is always the same.

We consider the $r$-variable $r$-index polynomial sets

$$
\left\{p_{n_{1}, \ldots, n_{r}}\left(x_{1}, \ldots, x_{r}\right)\right\} \equiv\left\{p_{n_{1}, \ldots, n_{r}}\left(x_{1}, \ldots, x_{r}\right)\right\}_{\left(n_{1}, \ldots, n_{r}\right) \in \mathbf{N}_{0} \times \cdots \times \mathbf{N}_{0}}
$$

$n_{k}$ denoting the degree of the polynomial with respect to the variable $x_{k}(k=$ $1,2, \ldots, r)$.

The $r$-variable monomiality principle is defined as follows.

Definition 7.1. An $r$-variable $r$-index polynomial set $\left\{p_{n_{1}, \ldots, n_{r}}\left(x_{1}, \ldots, x_{r}\right)\right\}$ is said to be quasi-monomial if $2 r$ operators, independent of $n_{1}, n_{2}, \ldots, n_{r}$, 
denoted by $\hat{P}_{x_{1}}, \ldots, \hat{P}_{x_{r}}, \hat{M}_{x_{1}}, \ldots, \hat{M}_{x_{r}}$, exist in such a way that

$$
\begin{aligned}
& \left\{\begin{array}{l}
\hat{P}_{x_{1}} p_{n_{1}, \ldots, n_{r}}\left(x_{1}, \ldots, x_{r}\right)=n_{1} p_{n_{1}-1, n_{2}, \ldots, n_{r}}\left(x_{1}, \ldots, x_{r}\right) \\
\ldots \ldots \ldots \ldots \ldots \ldots \ldots \ldots \ldots \ldots \ldots \ldots \ldots \ldots \ldots \ldots \ldots \\
\hat{P}_{x_{r}} p_{n_{1}, \ldots, n_{r}}\left(x_{1}, \ldots, x_{r}\right)=n_{r} p_{n_{1}, \ldots, n_{r-1}, n_{r}-1}\left(x_{1}, \ldots, x_{r}\right)
\end{array}\right. \\
& \left\{\begin{array}{l}
\hat{M}_{x_{1}} p_{n_{1}, \ldots, n_{r}}\left(x_{1}, \ldots, x_{r}\right)=p_{n_{1}+1, n_{2}, \ldots, n_{r}}\left(x_{1}, \ldots, x_{r}\right) \\
\ldots \ldots \ldots \ldots \ldots \ldots \ldots \ldots \ldots \ldots \ldots \ldots \ldots \ldots \ldots \\
\hat{M}_{x_{r}} p_{n_{1}, \ldots, n_{r}}\left(x_{1}, \ldots, x_{r}\right)=p_{n_{1}, \ldots, n_{r-1}, n_{r}+1}\left(x_{1}, \ldots, x_{r}\right) .
\end{array}\right.
\end{aligned}
$$

From the above formulas it follows that

$$
\left[\hat{P}_{x_{1}}, \hat{M}_{x_{1}}\right]=1, \ldots,\left[\hat{P}_{x_{r}}, \hat{M}_{x_{r}}\right]=1
$$

Under the above hypotheses, the main properties of the polynomial set under consideration can be easily derived, since

- If the derivative and multiplication operators have a differential realization, then

$$
\begin{aligned}
& \hat{M}_{x_{1}} \hat{P}_{x_{1}} p_{n_{1}, \ldots, n_{r}}\left(x_{1}, \ldots, x_{r}\right)=n_{1} p_{n_{1}, \ldots, n_{r}}\left(x_{1}, \ldots, x_{r}\right) \\
& \ldots \ldots \ldots \ldots \ldots \ldots \ldots \ldots \ldots \ldots \ldots \ldots \ldots \ldots \\
& \hat{M}_{x_{r}} \hat{P}_{x_{r}} p_{n_{1}, \ldots, n_{r}}\left(x_{1}, \ldots, x_{r}\right)=n_{r} p_{n_{1}, \ldots, n_{r}}\left(x_{1}, \ldots, x_{r}\right)
\end{aligned}
$$

i.e., we find $r$ (independent) differential equations satisfied by a polynomial set.

- Assuming $p_{0, \ldots, 0}\left(x_{1}, \ldots, x_{r}\right) \equiv 1$, an explicit expression of

$$
\left\{p_{n_{1}, \ldots, n_{r}}\left(x_{1}, \ldots, x_{r}\right)\right\}
$$

is given by

$$
p_{n_{1}, \ldots, n_{r}}\left(x_{1}, \ldots, x_{r}\right)=\hat{M}_{x_{1}}^{n_{1}} \cdots \hat{M}_{x_{r}}^{n_{r}}(1) .
$$

- The exponential generating function of $\left\{p_{n_{1}, \ldots, n_{r}}\left(x_{1}, \ldots, x_{r}\right)\right\}$, assuming again $p_{0, \ldots, 0}\left(x_{1}, \ldots, x_{r}\right) \equiv 1$, is given by

$$
\begin{aligned}
e^{t_{1} \hat{M}_{x_{1}}+\cdots+t_{r} \hat{M}_{x_{r}}}(1) & =\sum_{n_{1}=0}^{\infty} \cdots \sum_{n_{r}=0}^{\infty} \frac{t_{1}^{n_{1}}}{n_{1} !} \frac{\left(t_{1} \hat{M}_{x_{1}}\right)^{n_{1}} \cdots\left(t_{r} \hat{M}_{x_{r}}\right)^{n_{r}}}{n_{1} ! \cdots n_{r} !} \\
& =\sum_{n_{1}=0}^{\infty} \cdots \sum_{n_{r}=0}^{\infty} \frac{t_{1}^{n_{1}}}{n_{1} !} \cdots \frac{t_{r}^{n_{r}}}{n_{r} !} p_{n_{1}, \ldots, n_{r}}\left(x_{1}, \ldots, x_{r}\right) .
\end{aligned}
$$

Theorem 7.1. Let $\hat{A}_{1}, \ldots, \hat{A}_{r}$, be commuting operators (i.e., $\left[\hat{A}_{i}, \hat{A}_{j}\right]=0$, $\forall i, j)$ independent of the parameters $t_{1}, \ldots, t_{r}$. Then the Hausdorff identity 
holds:

$$
\begin{aligned}
e^{t_{1} \hat{A}_{1}+\cdots+t_{r} \hat{A}_{r}} & \hat{C} e^{-t_{1} \hat{A}_{1}-\cdots-t_{r} \hat{A}_{r}} \\
=\hat{C}+ & \left(\sum_{i=0}^{r} t_{i}\left[\hat{A}_{i}, \hat{C}\right]\right)+\frac{1}{2 !}\left(\sum_{i, j=0}^{r} t_{i} t_{j}\left[\hat{A}_{i},\left[\hat{A}_{j}, \hat{C}\right]\right]\right) \\
& +\frac{1}{3 !}\left(\sum_{i, j, k=0}^{r} t_{i} t_{j} t_{k}\left[\hat{A}_{i},\left[\hat{A}_{j},\left[\hat{A}_{k}, \hat{C}\right]\right]\right]\right)+\cdots
\end{aligned}
$$

Theorem 7.2. Consider $r$ operators $\hat{\Phi}_{x_{1}}, \ldots, \hat{\Phi}_{x_{r}}$ commuting respectively with $\hat{P}_{x_{1}}, \ldots, \hat{P}_{x_{r}}$ and such that

$$
e^{t_{1} \hat{\Phi}_{x_{1}}+\cdots+t_{r} \hat{\Phi}_{x_{r}}}(1)=1 .
$$

Put

$$
Q_{n_{1}, \ldots, n_{r}}\left(x_{1}, \ldots, x_{r} ; t_{1}, \ldots, t_{r}\right):=e^{t_{1} \hat{\Phi}_{x_{1}}+\cdots+t_{r} \hat{\Phi}_{x_{r}}} p_{n_{1}, \ldots, n_{r}}\left(x_{1}, \ldots, x_{r}\right),
$$

and suppose that there exist $r$ operators $\hat{M}_{1, x_{1}}\left(t_{1}, \ldots, t_{r}\right), \ldots, \hat{M}_{1, x_{r}}\left(t_{1}, \ldots, t_{r}\right)$ such that

$Q_{n_{1}, \ldots, n_{r}}\left(x_{1}, \ldots, x_{r} ; t_{1}, \ldots, t_{r}\right)=\left(\hat{M}_{1, x_{1}}\left(t_{1}, \ldots, t_{r}\right)\right)^{n_{1}} \cdots\left(\hat{M}_{1, x_{r}}\left(t_{1}, \ldots, t_{r}\right)\right)^{n_{r}}$

and, furthermore, $\forall t_{1}, \ldots, t_{r}$

$$
\left[\hat{P}_{x_{1}}, \hat{M}_{1, x_{1}}\left(t_{1}, \ldots, t_{r}\right)\right]=\cdots=\left[\hat{P}_{x_{r}}, \hat{M}_{1, x_{r}}\left(t_{1}, \ldots, t_{r}\right)\right]=1 .
$$

Then the polynomial set $Q_{n_{1}, \ldots, n_{r}}\left(x_{1}, \ldots, x_{r} ; t_{1}, \ldots, t_{r}\right)$ is quasi-monomial with respect to the operators $\hat{P}_{x_{1}}, \ldots \hat{P}_{x_{r}}, \hat{M}_{1, x_{1}}\left(t_{1}, \ldots, t_{r}\right), \ldots, \hat{M}_{1, x_{r}}\left(t_{1}, \ldots, t_{r}\right)$.

Theorem 7.3. Consider the polynomial set $\left\{p_{n_{1}, \ldots, n_{r}}\left(x_{1}, \ldots, x_{r}\right)\right\}$ putting again $p_{0, \ldots, 0}\left(x_{1}, \ldots, x_{r}\right) \equiv 1$, and suppose that it is quasi-monomial with respect to the operators $\hat{P}_{x_{1}}, \ldots, \hat{P}_{x_{r}}, \hat{M}_{x_{1}}, \ldots, \hat{M}_{x_{r}}$. Let $\hat{\Phi}_{x_{1}}, \ldots, \hat{\Phi}_{x_{r}}$ be operators independent of the parameters $t_{1}, \ldots, t_{r}$ and such that

$$
\left[\hat{\Phi}_{x_{1}}, \hat{P}_{x_{1}}\right]=\cdots=\left[\hat{\Phi}_{x_{r}}, \hat{P}_{x_{r}}\right]=0, \quad e^{t_{1} \hat{\Phi}_{x_{1}}+\cdots+t_{r} \hat{\Phi}_{x_{r}}}(1)=1 .
$$

Define again the polynomial set

$$
Q_{n_{1}, \ldots, n_{r}}\left(x_{1}, \ldots, x_{r} ; t_{1}, \ldots, t_{r}\right):=e^{t_{1} \hat{\Phi}_{x_{1}}+\cdots+t_{r} \hat{\Phi}_{x_{r}}} p_{n_{1}, \ldots, n_{r}}\left(x_{1}, \ldots, x_{r}\right) .
$$

Then the multiplication operators are given by the Hausdorff expansions:

$$
\begin{aligned}
& \hat{M}_{1, x_{1}}=\hat{M}_{x_{1}}+\left\{t_{1}\left[\hat{\Phi}_{x_{1}}, \hat{M}_{x_{1}}\right]+\cdots+t_{r}\left[\hat{\Phi}_{x_{r}}, \hat{M}_{x_{1}}\right]\right\} \\
& +\frac{1}{2 !}\left(\sum_{i, j=0}^{r} t_{i} t_{j}\left[\hat{\Phi}_{x_{i}},\left[\Phi_{x_{j}}, \hat{M}_{x_{1}}\right]\right]\right)+\cdots \\
& \hat{M}_{1, x_{r}}=\hat{M}_{x_{r}}+\left\{t_{1}\left[\hat{\Phi}_{x_{1}}, \hat{M}_{x_{r}}\right]+\cdots+t_{r}\left[\hat{\Phi}_{x_{r}}, \hat{M}_{x_{r}}\right]\right\}
\end{aligned}
$$




$$
+\frac{1}{2 !}\left(\sum_{i, j=0}^{r} t_{i} t_{j}\left[\hat{\Phi}_{x_{i}},\left[\Phi_{x_{j}}, \hat{M}_{x_{r}}\right]\right]\right)+\cdots
$$

\section{ACKNOWLEDGEMENTS}

This article was concluded in the framework of the Italian National Group for Scientific Computation (G.N.C.S.).

\section{REFERENCES}

1. G. Dattoli, Hermite-Bessel and Laguerre-Bessel functions: a by-product of the monomiality principle. Advanced special functions and applications (Melfi, 1999), 147-164, Proc. Melfi Sch. Adv. Top. Math. Phys., 1, Aracne, Rome, 2000.

2. A. BERNARDini and P. E. RiCCI, A constructive approach to the monomiality operators. South East Asian J. Math. Math. Sci. 3(2005), No. 3, 33-44.

3. A. Bernardini and P. E. Ricci, A note on the multi-variable Gould-Hopper and Laguerre polynomials. J. Comput. Anal. Appl. (to appear).

4. P. Appell and J. KAmpé De FÉRIet, Fonctions hypergéométriques et hypersphériques. Polynômes d'Hermite. Gauthier-Villars, Paris, 1926.

5. H. W. Gould and A. T. Hopper, Operational formulas connected with two generalizations of Hermite polynomials. Duke Math. J. 29(1962), 51-63.

6. H. M. SRIVAstava and H. L. Manocha, A treatise on generating functions. Ellis Horwood Series: Mathematics and its Applications. Ellis Horwood Ltd., Chichester; Halsted Press [John Wiley \& Sons, Inc.], New York, 1984.

7. J. F. Steffensen, The poweroid, an extension of the mathematical notion of power. Acta Math. 73(1941), 333-366.

8. Y. Ben Cheikh, Some results on quasi-monomiality. Proc. Workshop "Advanced Special Functions and Related Topics in Differential Equations", Melfi, June 24-29, 2001; Appl. Math. Comput. 141(2003), No. 1, 63-76.

9. Y. Ben CheIkn, On obtaining dual sequences via quasi-monomiality. Georgian Math. J. 9(2002), No. 3, 413-422.

10. G. Dattoli, P. L. Ottaviani, A. Torre, and L. VÁzquez, Evolution operator equations: integration with algebraic and finite-difference methods. Applications to physical problems in classical and quantum mechanics and quantum field theory. Riv. Nuovo Cimento Soc. Ital. Fis. (4) 20(1997), No. 2, 133 pp.

11. G. Dattoli, H. M. Srivastava, and P. E. Ricci, Two-index multidimensional Gegenbauer polynomials and their integral representations. Math. Comput. Modelling 37(2003), No. 3-4, 283-291.

12. G. Dattoli, S. Lorenzutta, P. E. Ricci, and C. Cesarano, On a family of hybrid polynomials. Integral Transforms Spec. Funct. 15(2004), No. 6, 485-490.

13. G. Bretti and P. E. Ricci, Multidimensional extensions of the Bernoulli and Appell polynomials. Taiwanese J. Math. 8(2004), No. 3, 415-428.

14. R. M. Wilcox, Exponential operators and parameter differentiation in quantum physics. J. Mathematical Phys. 8(1967), 962-982.

15. A. Bernardini, G. Dattoli, and P. E. Ricci, L-exponentials and higher order Laguerre polynomials. Proceedings of the Fourth International Conference of the Society for Special Functions and their Applications (SSFA), 13-26, Soc. Spec. Funct. Appl., Chennai, 2003. 
16. O. V. Viskov, A commutative-like noncommutative identity. Acta Sci. Math. (Szeged) 59(1994), No. 3-4, 585-590.

(Received 13.07.2006)

Authors' addresses:

C. Belingeri

Università di Roma "La Sapienza"

Dipartimento di Metodi e Modelli Matematici

per le Scienze Applicate

Via A. Scarpa, 14 - 00161 Roma, Italia

E-mail: belingeri@dmmm.uniroma1.it

G. Dattoli

ENEA - Unità Tecnico Scientifica Tecnologie Fisiche Avanzate

Centro Ricerche Frascati

C.P. 65 - 00044 Frascati (Roma), Italia

E-mail: dattoli@frascati.enea.it

P. E. Ricci

Università di Roma "La Sapienza"

Dipartimento di Matematica

P.le A. Moro, 2, 00185 Roma, Italia

E-mail: Paoloemilio.Ricci@uniroma1.it 